\title{
Preparation and Characterization of ZnO Hollow Spheres and ZnO- carbon Composite Materials Using Colloidal Carbon Spheres as Templates
}

\author{
Xi Wang ${ }^{\dagger, \ddagger}$, Peng $\mathrm{Hu}^{\dagger, \ddagger}$, Yuan Fangli ${ }^{\dagger, *}$, Lingjie $\mathrm{Yu}^{\dagger, \neq}$ \\ † State Key Laboratory of Multi-phase Complex System, Institute of Process Engineering, \\ Chinese Academy of Sciences, Beijing 100080, China \\ ‡ Graduate School of the Chinese Academy of Sciences, Beijing 100049, China
}

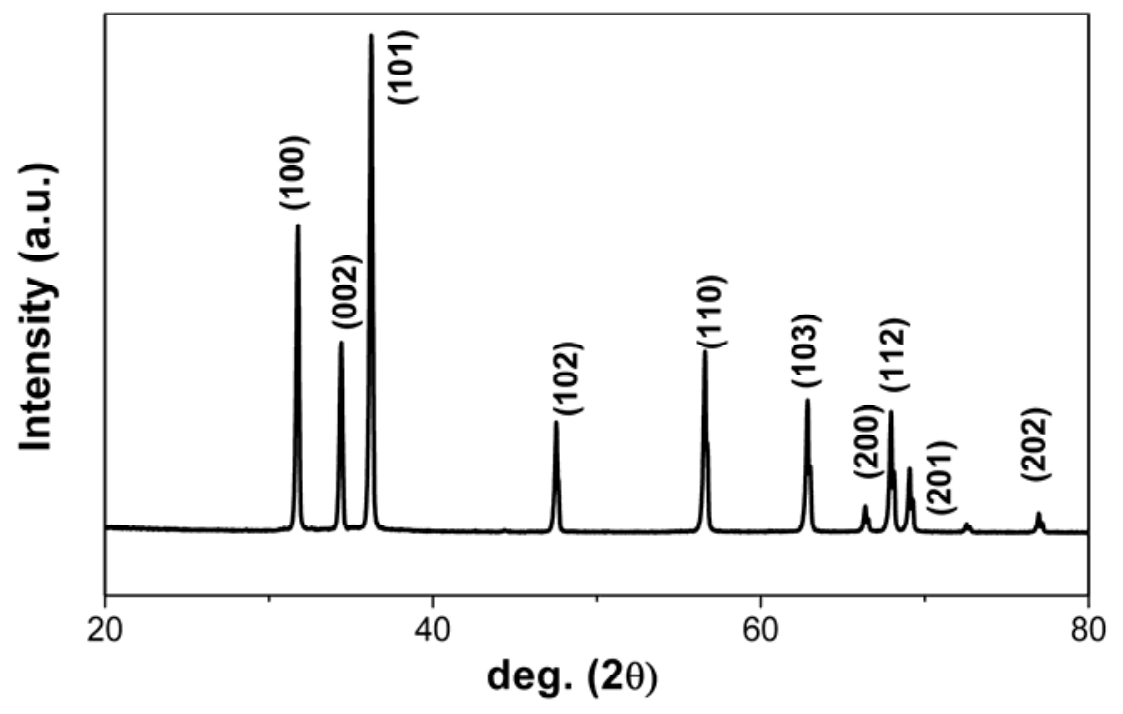

Figure S1 XRD pattern of ZnO hollow spheres 


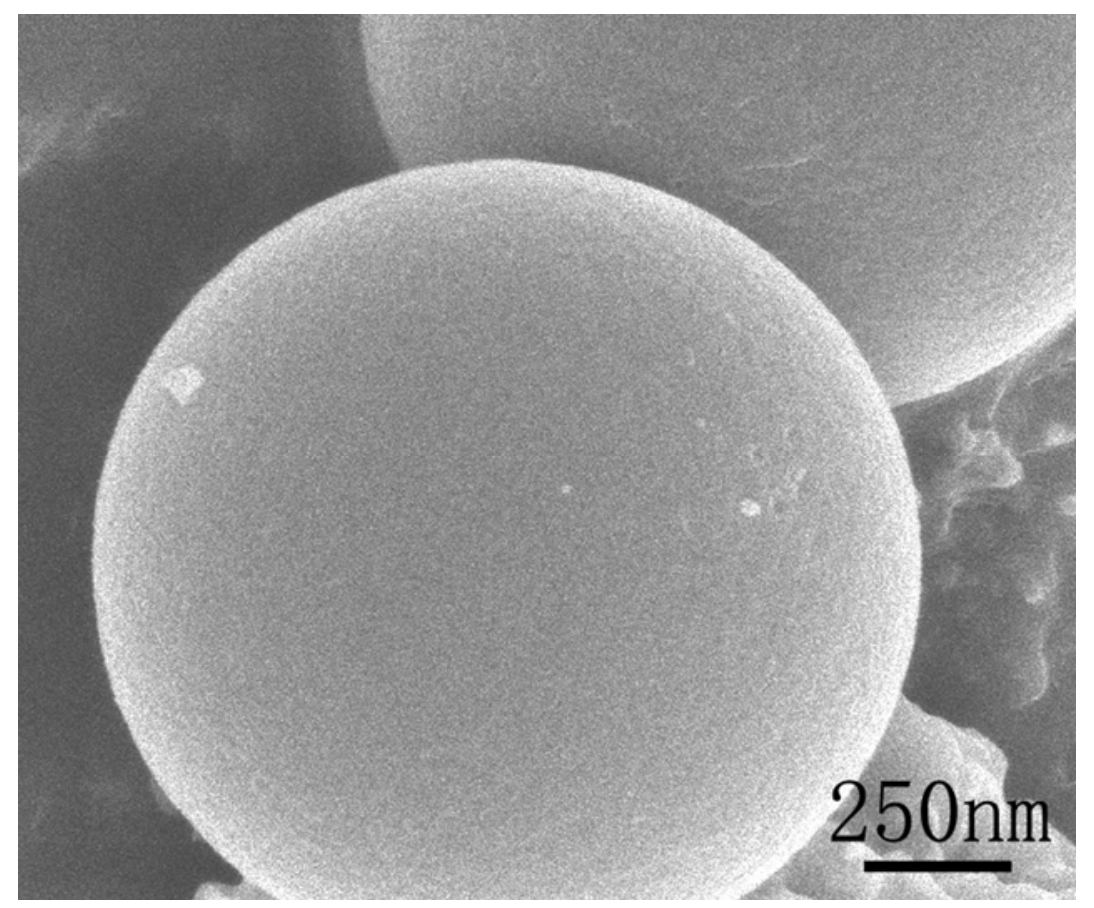

Figure S2. Detailed surface structure of individual CCSs.

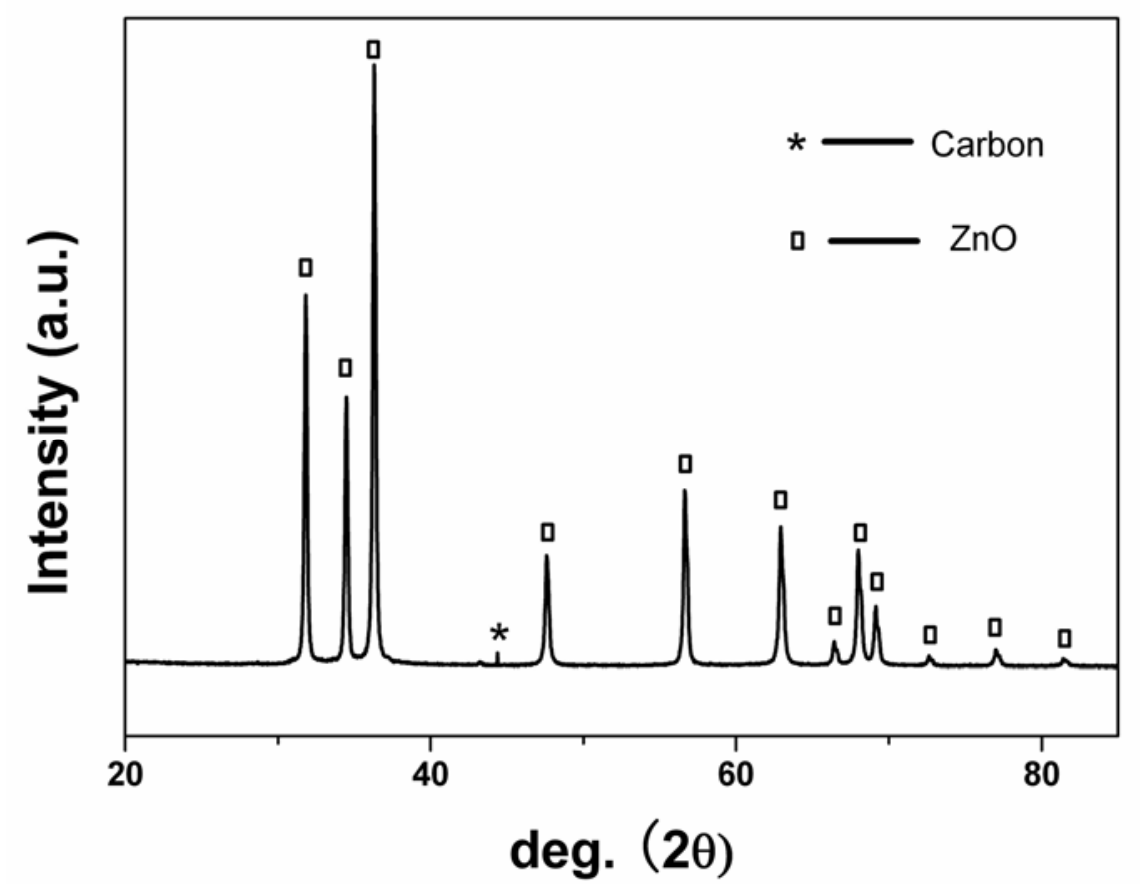

Figure S3. XRD patterns of CCSs@ZnO nanoparticles. 


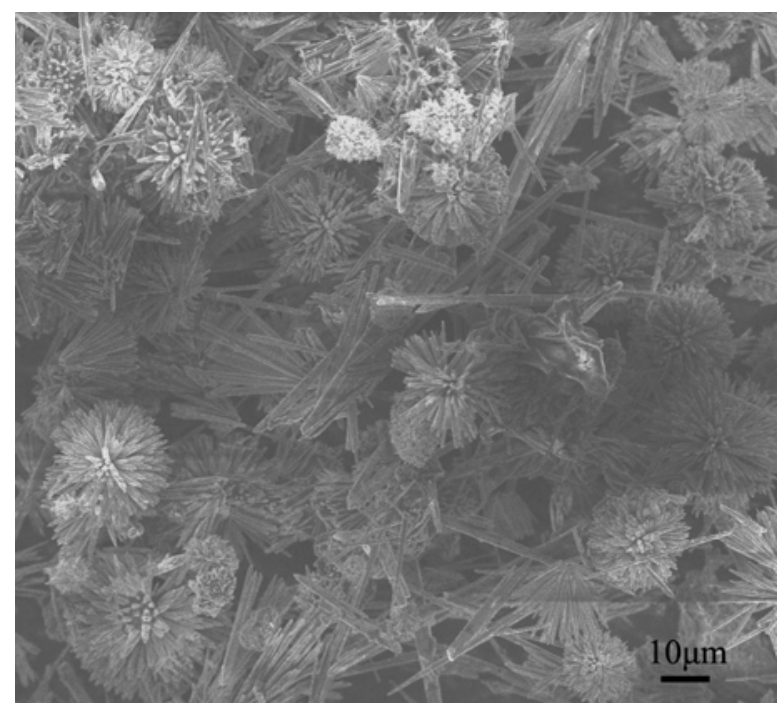

Figure S4. High yields of ZnO-carbon dandelions (SEM image).



Figure S5. XRD pattern of ZnO-carbon composite dandelions. 


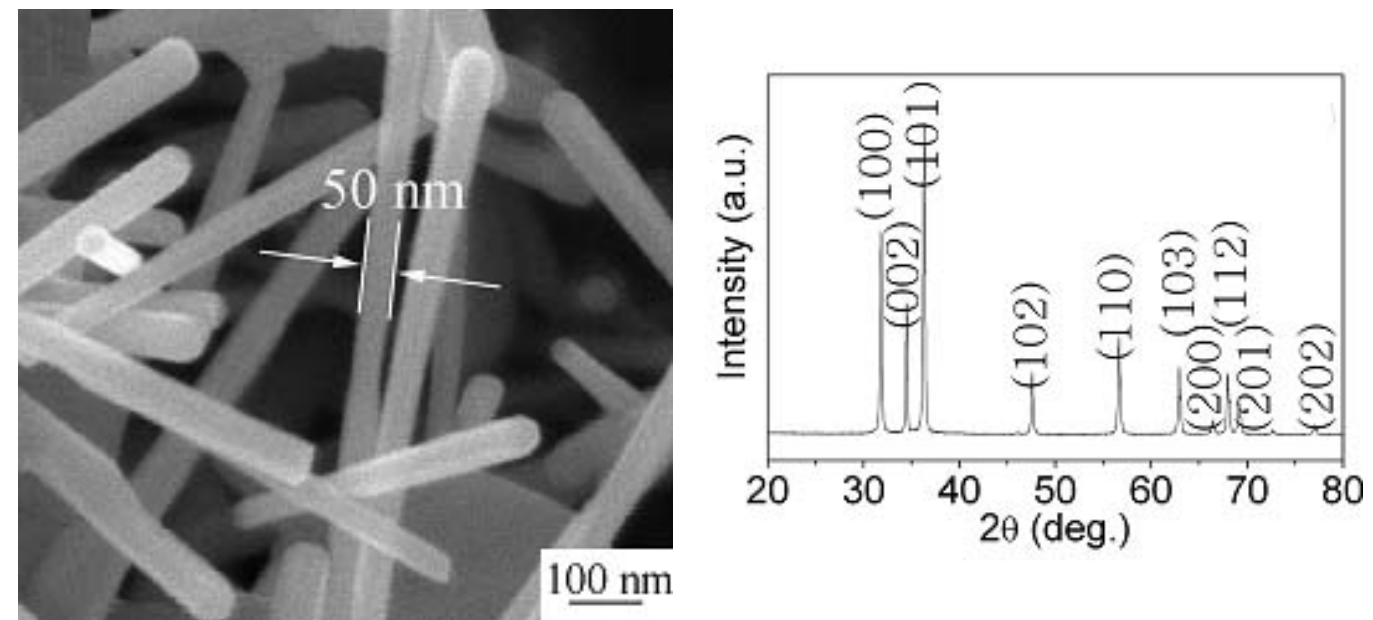

Figure S5 SEM image and XRD spectrum of ZnO nanorods synthesized by plasma 